\title{
Cost-Minimization Analysis of Dexmedetomidine Compared to Other Sedatives for Short-Term Sedation During Mechanical Ventilation in the United States [Corrigendum]
}

Aggarwal J, Lustrino J, Stephens J, Morgenstern D, Tang WY. Clinicoecon Outcomes Res. 2020;12:389-397.
Tables 1-4 on pages 391-394, the reference citations listed in the Data Source columns are incorrect. The correct Tables $1-4$ are shown below.

Table I Key Model Input Parameters

\begin{tabular}{|c|c|c|c|c|}
\hline Parameter & Dexmedetomidine & Propofol & Midazolam & Data Source \\
\hline Patient weight $(\mathrm{kg})$ & 82.5 & 82.5 & 82.5 & $\begin{array}{l}\text { Average weight of an adult in the US, Centers for } \\
\text { Disease Control and Prevention }{ }^{22}\end{array}$ \\
\hline Length of stay in ICU (days) & 1.9 & 3.0 & 3.0 & Maldonado et al ${ }^{18}$ \\
\hline Duration of MV (days) & 0.496 & 0.463 & 0.529 & Intubation time, Maldonado et al ${ }^{18}$ \\
\hline \multicolumn{5}{|l|}{ Sedative } \\
\hline Treatment duration (hours) & 13 & 11 & 10 & Maldonado et $\mathrm{al}^{18}$ \\
\hline$\%$ that receive loading dose $(\%)$ & 100 & 0 & 0 & Assumption based on study protocol, Maldonado et al ${ }^{18}$ \\
\hline Loading dose $(\mu \mathrm{g} / \mathrm{kg})$ & 0.4 & - & - & \\
\hline Duration of loading dose (min) & 10 & - & - & Assumption based on prescribing information ${ }^{24}$ \\
\hline $\begin{array}{l}\text { Maintenance dose }(\mu g / \mathrm{kg} / \mathrm{hr} \\
\text { or } \mathrm{mg} / \mathrm{kg} / \mathrm{hr})^{\mathrm{a}}\end{array}$ & 0.35 & 1.578 & 0.018 & $\begin{array}{l}\text { Calculated dose for midazolam based on average patient } \\
\text { weight used in model, Maldonado et } \text { al }^{18}\end{array}$ \\
\hline $\begin{array}{l}\text { Time to prepare one bag of } \\
\text { sedative (seconds) }\end{array}$ & 35 & - & 35 & $\begin{array}{l}\text { Assumption based on time to prepare one emergency } \\
\text { syringe, Fraind et } \mathrm{al}^{27} \text { and Jelacic et } \mathrm{al}^{41}\end{array}$ \\
\hline \multicolumn{5}{|l|}{ Pain medication } \\
\hline Total dose of morphine (mg) & 50.3 & 51.6 & 122.5 & Maldonado et al $^{18}$ \\
\hline \multicolumn{5}{|l|}{ Adverse events } \\
\hline Occurrence of delirium (\%) & 10.0 & 44.4 & 42.5 & ITT population, Maldonado et al ${ }^{18}$ \\
\hline
\end{tabular}

Notes: ${ }^{\mathrm{a} D e x m e d e t o m i d i n e: ~} \mu \mathrm{g} / \mathrm{kg} / \mathrm{hr}$; propofol and midazolam: $\mathrm{mg} / \mathrm{kg} / \mathrm{hr} ;{ }^{\mathrm{b}} 200 \mu \mathrm{g} / \mathrm{bag}$ of dexmedetomidine; $50 \mathrm{mg} / \mathrm{bag}$ of midazolam.

Abbreviations: ICU, intensive care unit; ITT, intention-to-treat; LOS, length of stay; MV, mechanical ventilation; US, United States.

Table 2 Cost Inputs

\begin{tabular}{|l|l|l|}
\hline Variables & Cost (2018) & Data Source \\
\hline ICU room and board, cost per day & & \\
Day I & $\$ 11,421.91$ & ${\text { Dasta et } \mathrm{al}^{3}}^{3}$ \\
Day 2 & $\$ 5989.35$ & Dasta et al $^{3}$ \\
Day 3+ & $\$ 5454.83$ & Dasta et al $^{3}$ \\
\hline $\begin{array}{l}\text { MV, cost per day } \\
\text { Day I }\end{array}$ & $\$ 7070.38$ & \\
\hline
\end{tabular}

(Continued)

submit your manuscript | www.dovepress.com
DovePress
http://doi.org// 0.21 4 47/CEOR.S284346


Table 2 (Continued).

\begin{tabular}{|c|c|c|}
\hline Variables & Cost (2018) & Data Source \\
\hline Day 2 & $\$ 2227.16$ & Dasta et $\mathrm{al}^{3}$ \\
\hline Day $3+$ & $\$ 1343.15$ & Dasta et $\mathrm{al}^{3}$ \\
\hline \multicolumn{3}{|l|}{ Sedative preparation } \\
\hline Pharmacist hourly rate & $\$ 55.23$ & Society of Critical Care Medicine ${ }^{26}$ \\
\hline \multicolumn{3}{|l|}{ Respiratory monitoring costs } \\
\hline Arterial blood gases, per ICU day & $\$ 26.07$ & $\begin{array}{l}\text { CPT 82,803, Blood gases any } \\
\text { combination, } \mathrm{CMS}^{28}\end{array}$ \\
\hline Physician consultation cost, per ICU day & $\$ 226.80$ & $\begin{array}{l}\text { CPT 99,29I, Critical care first } 30-74 \\
\text { minutes, } \text { CMS }^{29}\end{array}$ \\
\hline Tracheal intubation cost, per ICU stay & $\$ 155.52$ & $\begin{array}{l}\text { CPT } 31,730 \text {, Introduction of } \\
\text { indwelling tube for oxygen therapy }{ }^{29}\end{array}$ \\
\hline \multicolumn{3}{|l|}{ Toxicology testing } \\
\hline $\begin{array}{l}\text { Benzodiazepine testing cost, per ICU treatment day if primary sedative was } \\
\text { midazolam, per ICU stay if midazolam is administered for rescue sedation only }\end{array}$ & $\$ 71.83$ & $\begin{array}{l}\text { CPT } 80,307 \text {, Drug test by chemistry } \\
\text { analyzers }^{28}\end{array}$ \\
\hline \multicolumn{3}{|l|}{ Medication costs (wholesale acquisition cost per vial) } \\
\hline Dexmedetomidine & $\$ 42.00$ & $200 \mu \mathrm{g}$ vial, Truven Health Analytics ${ }^{30}$ \\
\hline Midazolam & $\$ 1.92$ & $50 \mathrm{mg}$ vial, Truven Health Analytics ${ }^{30}$ \\
\hline Midazolam & $\$ 0.55$ & $\begin{array}{l}5 \mathrm{mg} \text { vial for rescue sedation, Truven } \\
\text { Health Analytics }{ }^{30}\end{array}$ \\
\hline Propofol & $\$ 3.88$ & $\begin{array}{l}500 \mathrm{mg} \text { vial, Truven Health } \\
\text { Analytics }^{30}\end{array}$ \\
\hline Morphine & $\$ 7.05$ & $\begin{array}{l}250 \mathrm{mg} \text { vial, Truven Health } \\
\text { Analytics }^{30}\end{array}$ \\
\hline Atropine, $0.5 \mathrm{mg}$ per bradycardia event & $\$ 1.61$ & I mg vial, Truven Health Analytics ${ }^{30}$ \\
\hline Haloperidol, 10 mg per delirium event & $\$ 0.88$ & $5 \mathrm{mg}$ vial, Truven Health Analytics ${ }^{30}$ \\
\hline Labetalol, $20 \mathrm{mg}$ per hypertension event & $\$ 3.35$ & $\begin{array}{l}100 \text { mg vial, Truven Health } \\
\text { Analytics }^{30}\end{array}$ \\
\hline Norepinephrine, $4 \mathrm{mg}$ per hypotension event & $\$ 4.60$ & 4 mg vial, Truven Health Analytics ${ }^{30}$ \\
\hline Cefazolin, I g per infection event & $\$ 6.33$ & $2 \mathrm{~g}$ vial, Truven Health Analytics ${ }^{30}$ \\
\hline
\end{tabular}

Abbreviations: CMS, Centers for Medicare and Medicaid Services; CPT, current procedural code; ICU, intensive care unit; MV, mechanical ventilation.

Table 3 Sensitivity Analysis Clinical Inputs for Propofol Comparison

\begin{tabular}{|c|c|c|c|c|c|}
\hline \multirow[t]{2}{*}{ Parameters } & \multicolumn{2}{|c|}{$\begin{array}{l}\text { Dexmedetomidine } \\
\text { Arm }\end{array}$} & \multicolumn{2}{|c|}{ Propofol Arm } & \multirow[t]{2}{*}{ Data Source } \\
\hline & $\begin{array}{l}\text { Lower } \\
\text { Value }\end{array}$ & $\begin{array}{l}\text { Upper } \\
\text { Value }\end{array}$ & $\begin{array}{l}\text { Lower } \\
\text { Value }\end{array}$ & $\begin{array}{l}\text { Upper } \\
\text { Value }\end{array}$ & \\
\hline Length of stay in ICU (days) & 0.96 & 1.90 & 0.96 & 3.00 & Corbett et al $^{32}$ Maldonado et al ${ }^{18}$ \\
\hline Duration of MV (days) & 0.23 & 0.50 & 0.25 & 0.54 & Djaiani et al, ${ }^{14}$ Srivastava et al ${ }^{15}$ \\
\hline $\begin{array}{l}\text { Sedative } \\
\text { Treatment duration relative to } \\
\text { MV duration ( } \pm \text { hours) })^{\mathrm{a}}\end{array}$ & -0.59 & 6.00 & -0.44 & -0.10 & Herr et al, ${ }^{19}$ Maldonado et al, ${ }^{18}$ Srivastava et al ${ }^{15}$ \\
\hline$\%$ that receive loading dose (\%) & 90 & 100 & 0 & 100 & $\begin{array}{l}\text { Dexmedetomidine inputs did not vary in published literature, } \\
\text { varied by } 10 \% \text { for analysis. Corbett et al, }{ }^{32} \text { Djaiani et al, }{ }^{14} \\
\text { Herr et al, }{ }^{19} \text { Maldonado et al, }{ }^{18} \text { Srivastava et al }{ }^{15}\end{array}$ \\
\hline
\end{tabular}

(Continued) 
Table 3 (Continued).

\begin{tabular}{|c|c|c|c|c|c|}
\hline \multirow[t]{2}{*}{ Parameters } & \multicolumn{2}{|c|}{$\begin{array}{l}\text { Dexmedetomidine } \\
\text { Arm }\end{array}$} & \multicolumn{2}{|c|}{ Propofol Arm } & \multirow[t]{2}{*}{ Data Source } \\
\hline & $\begin{array}{l}\text { Lower } \\
\text { Value }\end{array}$ & $\begin{array}{l}\text { Upper } \\
\text { Value }\end{array}$ & $\begin{array}{l}\text { Lower } \\
\text { Value }\end{array}$ & $\begin{array}{l}\text { Upper } \\
\text { Value }\end{array}$ & \\
\hline Loading dose $(\mu \mathrm{g} / \mathrm{kg}$ or $\mathrm{mg} / \mathrm{kg} /$ & 0.40 & 1.00 & 0.00 & 4.00 & Corbett et al, ${ }^{32}$ Djaiani et al, ${ }^{14}$ Herr et al, ${ }^{19}$ \\
\hline hr)a & & & & & Maldonado et al, ${ }^{18}$ Srivastava et al ${ }^{15}$ \\
\hline $\begin{array}{l}\text { Duration of loading dose } \\
\text { (minutes)a }\end{array}$ & 10.00 & 20.00 & 0.00 & 15.00 & Herr et al, ${ }^{19}$ Maldonado et al, ${ }^{18}$ Srivastava et al ${ }^{15}$ \\
\hline $\begin{array}{l}\text { Maintenance dose }(\mu g / k g / h r \\
\text { or } \mathrm{mg} / \mathrm{kg} / \mathrm{hr}) \mathrm{b}\end{array}$ & 0.31 & 0.55 & 0.04 & 2.00 & Corbett et al, ${ }^{32}$ Djaiani et al, ${ }^{14}$ Srivastava et al ${ }^{15}$ \\
\hline $\begin{array}{l}\text { Time to prepare bag }(200 \mu \mathrm{g}) \\
\text { of dexmedetomidine or vial of } \\
\text { propofol(seconds) }\end{array}$ & 25 & 115 & 0 & 59 & Fraind et al, ${ }^{27}$ Jelacic et al, ${ }^{41}$ van der Linden ${ }^{42}$ \\
\hline \multicolumn{6}{|l|}{ Rescue sedation, midazolam } \\
\hline $\begin{array}{l}\% \text { that receive rescue sedation } \\
\text { (\%) }\end{array}$ & 0 & 100 & 0 & 100 & Corbett et al, ${ }^{32}$ Maldonado et al, ${ }^{18}$ Srivastava et al ${ }^{15}$ \\
\hline Rescue sedative dose (mg) & 0 & 1.5 & 0 & 1 & Corbett et al, ${ }^{32}$ Maldonado et al, ${ }^{18}$ Srivastava et al ${ }^{15}$ \\
\hline \multicolumn{6}{|l|}{ Pain medication } \\
\hline $\begin{array}{l}\% \text { treated with pain medication } \\
\text { (\%) }\end{array}$ & 90 & 100 & 90 & 100 & $\begin{array}{l}\text { Inputs did not vary in published literature, varied by } 10 \% \text { for } \\
\text { analysis. Corbett et al, }{ }^{32} \text { Djaiani et al, }{ }^{14} \text { Herr et al, }{ }^{19} \\
\text { Maldonado et al, }{ }^{18} \text { Srivastava et al }{ }^{15}\end{array}$ \\
\hline Total dose of morphine (mg) & 2.95 & 50.30 & 6.00 & 51.60 & Corbett et al, ${ }^{32}$ Herr et al, ${ }^{19}$ Maldonado et al ${ }^{18}$ \\
\hline \multicolumn{6}{|l|}{ Adverse events } \\
\hline Occurrence of bradycardia (\%) & 0 & 3 & 0 & I & $\begin{array}{l}\text { Corbett et al, }{ }^{32} \text { Djaiani et al, }{ }^{14} \text { Herr et al, }{ }^{19} \text { Maldonado } \\
\text { et al, }{ }^{18} \text { Srivastava et al }{ }^{15}\end{array}$ \\
\hline Occurrence of delirium (\%) & 0 & 12 & 0 & 44 & $\begin{array}{l}\text { Corbett et al, }{ }^{32} \text { Djaiani et al, }{ }^{14} \text { Herr et al, }{ }^{19} \text { Maldonado } \\
\text { et al, }{ }^{18} \text { Srivastava et al }{ }^{15}\end{array}$ \\
\hline Occurrence of hypertension (\%) & 0 & 12 & 0 & 4 & $\begin{array}{l}\text { Corbett et al, }{ }^{32} \text { Djaiani et al, }{ }^{14} \text { Herr et al, }{ }^{19} \text { Maldonado } \\
\text { et al, }{ }^{18} \text { Srivastava et al }{ }^{15}\end{array}$ \\
\hline Occurrence of hypotension (\%) & 0 & 81 & 0 & 67 & $\begin{array}{l}\text { Corbett et al, }{ }^{32} \text { Djaiani et al, }{ }^{14} \text { Herr et al, }{ }^{19} \text { Maldonado } \\
\text { et al, }{ }^{18} \text { Srivastava et al }{ }^{15}\end{array}$ \\
\hline Occurrence of infection (\%) & 0 & 10 & 0 & 10 & $\begin{array}{l}\text { Inputs did not vary in published literature, varied by } 10 \% \text { for } \\
\text { analysis. Corbett et all, }{ }^{32} \text { Djaiani et al, }{ }^{14} \text { Herr et al, }{ }^{19} \\
\text { Maldonado et al, }{ }^{18} \text { Srivastava et al }{ }^{15}\end{array}$ \\
\hline
\end{tabular}

Notes: ${ }^{\mathrm{a} D e x m e d e t o m i d i n e: ~} \mu \mathrm{g} / \mathrm{kg}$; propofol: $\mathrm{mg} / \mathrm{kg} / \mathrm{hr}$; ${ }^{\mathrm{b}}$ Dexmedetomidine: $\mu \mathrm{g} / \mathrm{kg} / \mathrm{hr} ;$ propofol: $\mathrm{mg} / \mathrm{kg} / \mathrm{hr}$.

Abbreviations: ICU, intensive care unit; MV, mechanical ventilation.

Table 4 Sensitivity Analysis Clinical Inputs for Midazolam Comparison

\begin{tabular}{|l|l|l|l|l|l|}
\hline \multirow{2}{*}{ Parameters } & \multicolumn{2}{l|}{\begin{tabular}{l} 
Dexmedetomidine \\
\multicolumn{2}{l|}{ Arm }
\end{tabular}} & \multicolumn{2}{l|}{ Midazolam Arm } & Data Source \\
\cline { 2 - 6 } & $\begin{array}{l}\text { Lower } \\
\text { Value }\end{array}$ & $\begin{array}{l}\text { Upper } \\
\text { Value }\end{array}$ & $\begin{array}{l}\text { Lower } \\
\text { Value }\end{array}$ & $\begin{array}{l}\text { Upper } \\
\text { Value }\end{array}$ & \\
\hline Length of stay in ICU (days) & 1.90 & 2.73 & 3.00 & 4.23 & Azeem et al, $^{13}$ Maldonado et al $^{18}$ \\
\hline Duration of MV (days) & 0.19 & 0.50 & 0.52 & 0.54 & Azeem et al, ${ }^{13}$ Srivastava et al, ${ }^{15} \mathrm{Wan}^{\text {et al }}{ }^{16}$ \\
\hline
\end{tabular}

(Continued) 
Table 4 (Continued).

\begin{tabular}{|c|c|c|c|c|c|}
\hline \multirow[t]{2}{*}{ Parameters } & \multicolumn{2}{|c|}{$\begin{array}{l}\text { Dexmedetomidine } \\
\text { Arm }\end{array}$} & \multicolumn{2}{|c|}{ Midazolam Arm } & \multirow[t]{2}{*}{ Data Source } \\
\hline & $\begin{array}{l}\text { Lower } \\
\text { Value }\end{array}$ & $\begin{array}{l}\text { Upper } \\
\text { Value }\end{array}$ & $\begin{array}{l}\text { Lower } \\
\text { Value }\end{array}$ & $\begin{array}{l}\text { Upper } \\
\text { Value }\end{array}$ & \\
\hline \multicolumn{6}{|l|}{ Sedative } \\
\hline $\begin{array}{l}\text { Treatment duration relative to MV } \\
\text { duration ( } \pm \text { hours) }\end{array}$ & -0.59 & 2.80 & -2.70 & -0.80 & Maldonado et al, ${ }^{18}$ Srivastava et al, ${ }^{15}$ Wan et al ${ }^{16}$ \\
\hline$\%$ that receive loading dose $(\%)$ & $0 \%$ & $100 \%$ & $0 \%$ & $100 \%$ & $\begin{array}{l}\text { Azeem et al, }{ }^{13} \text { Maldonado et al, }{ }^{18} \text { Srivastava et al, }{ }^{15} \text { Wan } \\
\text { et al }{ }^{16}\end{array}$ \\
\hline Loading dose $(\mu \mathrm{g} / \mathrm{kg}$ or $\mathrm{mg} / \mathrm{kg}) \mathrm{a}$ & 0.00 & 1.00 & 0.00 & 0.04 & $\begin{array}{l}\text { Azeem et al, }{ }^{13} \text { Maldonado et al, }{ }^{18} \text { Srivastava et al, }{ }^{15} \text { Wan } \\
\text { et al }{ }^{16}\end{array}$ \\
\hline Duration of loading dose (min) & 0.00 & 15.00 & 0.00 & 15.00 & $\begin{array}{l}\text { Azeem et al, }{ }^{13} \text { Maldonado et al, }{ }^{18} \text { Srivastava et al, }{ }^{15} \text { Wan } \\
\text { et al }{ }^{16}\end{array}$ \\
\hline $\begin{array}{l}\text { Maintenance dose }(\mu \mathrm{g} / \mathrm{kg} / \mathrm{hr} \text { or } \mathrm{mg} / \\
\mathrm{kg} / \mathrm{hr}) \mathrm{b}\end{array}$ & 0.35 & 0.72 & 0.02 & 0.44 & Azeem et al, ${ }^{13}$ Maldonado et al, ${ }^{18}$ Wan et al ${ }^{16}$ \\
\hline $\begin{array}{l}\text { Time to prepare one bag of } \\
\text { sedative }(\mathrm{sec}) \mathrm{c}\end{array}$ & 25 & 115 & 25 & 115 & Fraind et al $^{27}$ Jelacic et al, ${ }^{41}$ van der Linden ${ }^{42}$ \\
\hline Rescue sedation, midazolam & & & & & $\begin{array}{l}\text { For the midazolam arm, considered in sensitivity analysis } \\
\text { for the primary sedative }\end{array}$ \\
\hline$\%$ that receive rescue sedation (\%) & 0 & 100 & - & - & $\begin{array}{l}\text { Azeem et al, }{ }^{13} \text { Maldonado et al, }{ }^{18} \text { Srivastava et al, }{ }^{15} \text { Wan } \\
\text { et al }{ }^{16}\end{array}$ \\
\hline Rescue sedative dose $(\mathrm{mg})$ & 0 & 6.7 & - & - & $\begin{array}{l}\text { Azeem et al, }{ }^{13} \text { Maldonado et al, }{ }^{18} \text { Srivastava et al, }{ }^{15} \text { Wan } \\
\text { et al }{ }^{16}\end{array}$ \\
\hline \multicolumn{6}{|l|}{ Pain medication } \\
\hline$\%$ treated with pain medication (\%) & 90 & 100 & 90 & 100 & $\begin{array}{l}\text { Inputs did not vary in published literature, varied by } 10 \% \\
\text { for analysis. Azeem et al, }{ }^{13} \text { Maldonado et al, }{ }^{18} \text { Srivastava } \\
\text { et al, }{ }^{15} \text { Wan et al }{ }^{16}\end{array}$ \\
\hline Total dose of morphine (mg) & 17.61 & 50.30 & 37.21 & 125.06 & Azeem et al, ${ }^{13}$ Maldonado et al, ${ }^{18}$ Wan et al ${ }^{16}$ \\
\hline \multicolumn{6}{|l|}{ Adverse events } \\
\hline Occurrence of bradycardia (\%) & 0 & 25 & 0 & 10 & $\begin{array}{l}\text { Azeem et al, }{ }^{13} \text { Maldonado et al, }{ }^{18} \text { Srivastava et al, }{ }^{15} \text { Wan } \\
\text { et al }{ }^{16}\end{array}$ \\
\hline Occurrence of delirium (\%) & 0 & 10 & 0 & 43 & Maldonado et al, ${ }^{18}$ Srivastava et al ${ }^{15}$ \\
\hline Occurrence of hypertension (\%) & 0 & 10 & 0 & 10 & $\begin{array}{l}\text { Inputs did not vary in published literature, varied by } 10 \% \\
\text { for analysis. Azeem et al, }{ }^{13} \text { Maldonado et al, }{ }^{18} \text { Srivastava } \\
\text { et al, }{ }^{15} \text { Wan et al }{ }^{16}\end{array}$ \\
\hline Occurrence of hypotension (\%) & 0 & 28 & 0 & 11 & $\begin{array}{l}\text { Azeem et al, }{ }^{13} \text { Maldonado et al, }{ }^{18} \text { Srivastava et al, }{ }^{15} \text { Wan } \\
\text { et al }{ }^{16}\end{array}$ \\
\hline Occurrence of infection (\%) & 0 & 10 & 0 & 10 & $\begin{array}{l}\text { Inputs did not vary in published literature, varied by } 10 \% \\
\text { for analysis. Azeem et all, }{ }^{13} \text { Maldonado et al, }{ }^{18} \text { Srivastava } \\
\text { et al, }{ }^{15} \text { Wan et al }{ }^{16}\end{array}$ \\
\hline
\end{tabular}

Notes: ${ }^{\mathrm{a} D e x m e d e t o m i d i n e: ~} \mu \mathrm{g} / \mathrm{kg}$; midazolam: $\mathrm{mg} / \mathrm{kg}$; ${ }^{\mathrm{b}}$ Dexmedetomidine: $\mu \mathrm{g} / \mathrm{kg} / \mathrm{hr} ;$ midazolam: $\mathrm{mg} / \mathrm{kg} / \mathrm{hr} ; \mathrm{c} 200 \mu \mathrm{g} / \mathrm{bag}$ of dexmedetomidine; $50 \mathrm{mg} / \mathrm{bag}$ of midazolam. Abbreviations: ICU, intensive care unit; MV, mechanical ventilation.

Page 397, references 41 and 42 are missing from the reference list. These references are shown below.

41. Jelacic S, Craddick K, Nair BG, et al. Relative Costs of Anesthesiologist Prepared, Hospital Pharmacy Prepared and Outsourced Anesthesia Drugs. Journal of Clinical Anesthesia. 2017;36:178-183.
42. van der Linden P, Douchamps J, Schmitt C, Forget D. Ready-to-Use Injection Preparations versus Conventional Reconstituted Admixtures: Economic Evaluation in a RealLife Setting. PharmacoEconomics. 2002;20(8):529-536. 


\section{Publish your work in this journal}

ClinicoEconomics and Outcomes Research is an international, peerreviewed open-access journal focusing on Health Technology Assessment, Pharmacoeconomics and Outcomes Research in the areas of diagnosis, medical devices, and clinical, surgical and pharmacologica intervention. The economic impact of health policy and health systems organization also constitute important areas of coverage. The manuscript management system is completely online and includes a very quick and fair peer-review system, which is all easy to use. Visit http://www.dovepress.com/testimonials.php to read real quotes from published authors. 